\title{
Is it Better to Defer the Radioactive Iodine Ablation in Patients with Differentiated Thyroid Cancer in the Current COVID 19 Pandemic?
}

Taalab $\mathrm{KH}^{*}$ and Abdulah B

El-Galaa Military Medical Campus, Military Medical Academy, Egypt
Research Article

Volume 3 Issue 2

Received Date: June 25, 2020

Published Date: July 09, 2020

DOI: $10.23880 /$ ijnmrs-16000129 gmail.com

\section{Abstract}

This study was conducted to highlight the negative impact of association of radioactive iodine-131 ablation therapy in patients with differentiated thyroid cancer with the pre-treatment hypothyroid status on corona virus disease-19 severity and prognosis.

Keywords: Covid 19; Differentiated Thyroid Cancer and Radioactive Iodine 131 Ablation Therapy

Abbreviations: SARS-CoV-2: Severe Acute Respiratory Syndrome Coronavirus 2; COVID-19: Coronavirus Disease-19; DTC: Differentiated Thyroid Carcinoma; RAI: Radioactive Iodine; Hb: Hemoglobin; WBC: White Blood Cell; Plt: Platelet.

\section{Introduction and Hypothesis}

An outbreak of an unknown infectious pneumonia has recently occurred and the pathogen of the disease was quickly identified as a novel corona virus (SARS-CoV-2, severe acute respiratory syndrome coronavirus 2), and the disease was named coronavirus disease-19 (COVID-19). Multiple mechanisms of CoV-2 might work together to cause lymphopenia and lymphocytes play a decisive role in maintaining immune homeostasis and inflammatory response throughout the body. Understanding the mechanism of diminished blood lymphocyte levels is expected to provide an effective strategy for the treatment of COVID-19. COVID-19 has been spreading in almost all the world countries and the virus has so far caused 55,233 confirmed cases and 2193 deaths in Egypt according to the reports of government.

The clinical manifestation of COVID-19 includes fever, cough, fatigue, myalgia, diarrhea and pneumonia, which can develop to acute respiratory distress syndrome, metabolic acidosis, and organ failure such as liver, kidney and heart; as well as coagulation dysfunction and septic shock. Unfortunately, there is no effective medication other than comprehensive support. However, the mild type of COVID-19 patients can recover shortly after appropriate clinical intervention [1]. The moderate type patients, especially the elderly/ ones with comorbidity; as cancer thyroid, can worsen and became more severe, indicating high morbidity and mortality rate. However, efficient indicators for disease severity, therapeutic response and disease outcome have not been fully investigated.

Routine examinations include complete blood count, coagulation profile, and serum biochemical test (including renal and liver function, creatine kinase, lactate dehydrogenase, and electrolytes). Complete blood count is the most available, efficient and economic examination. On the other hand; differentiated thyroid carcinoma (DTC) is the most common endocrine glands malignancy with relatively good prognosis and radioactive iodine (RAI) is considered effective for patients with total or nearly total thyroidectomy. The most common use of RAI is to destroy (called "to ablate") the small amount of normal thyroid that remains (called the "remnant") after surgery (near-total or total thyroidectomy) [2]. 


\section{This Therapy can be Used for Several Purposes:}

RAI ablation therapy in thyroid cancer patients is a safe treatment modality without any serious side effect; yet mild hematological side effects noted; a slightly depressed white blood cells (WBCs) count and also bone marrow hypoplasia have been observed in hypothyroid patients; the pre-ablation requested patient's status [3]. This study retrospects and analyzes the time-courses of complete blood count of cured and/or dead patients, in order to obtain key indicators of disease progression and outcome as well as to provide guidance for the sub-sequent clinical practice.

\section{Materials and Methods}

Twenty patients underwent near-/total thyroidectomy; all were of low risk; i.e. non-metastasized; ten patients were commenced on the suppressive therapy of Levo-thyroxine directly with postponing the RAI-131 ablation and the other ten received RAI-131 ablation therapy; then commenced 5-6 weeks later on the suppressive therapy; both groups almost similar in their demographic and histological data; selected after an interview in a highly dedicated center of thyroidology in our hospital; El-Galaa Military Medical Campus; GMMC; Cairo, Egypt; from November 2019 till June 2020.

We explain to them that postponing the ablation therapy via RAI131 for 6 months will not affect their disease prognosis and has the same efficiency depending on the long experience of more than thirty years of our center in dealing with similar cases and the main target might to reduce the potential risk of catching the Covid 19 infection; although not so high; from being in a hypothyroid status for nearly 4-6 weeks; administering a radioactive material with its temporary BM effects and the isolation stay for 3-5 nights in the hospital with its own risks itself. Ten patients decided to complete the pre-planned protocol of management (group A); whereas the other ten responded well to our recommendation of postponing the ablation therapy (group B).

Both groups were completely assured and followed up by phone and email. CBC results were available from the patients' medical records at the time of the ablation interview decision and at the 1st, 4th, and 6th months after. Three patients from the first group were Covid 19 infected through the six month time window of the current study whereas none of the second group [4].

\section{Results}

\section{In group $\mathrm{A}$}

Hemoglobin (Hb), white blood cell (WBC; especially lymphocytes) and platelet (Plt) values were significantly lower than baseline at 1 month after radioactive I131 ablative treatment; lymphocytes were less than $50 \%$ in the three Covid19 infected patients $(\mathrm{p}<0.0001)$; whereas none of the Covid19 negative seven patients developed similar lymphopenia. $\mathrm{Hb}$; platelet and WBC values were increased in all at the 4th. And 6th. Month; when Covid19 positive patients returned back negative by PCR9 (Table 1).

\begin{tabular}{|c|c|c|c|c|c|}
\hline Blood Cells & Gender & Descriptor & Before RAI131 & After RAI131 & Difference \\
\hline WBC, $\mathbf{1 0}^{\mathbf{9}} / \mathbf{L}$ & $\mathrm{M}$ & Mean \pm SD & $6.6 \pm 1.3$ & $5.6 \pm 1.1$ & $-1.0 \pm 0.2$ \\
\hline & F & Mean \pm SD & $6.0 \pm 1.3$ & $5.0 \pm 0.8$ & $-1.0 \pm 0.5$ \\
\hline Lymphocyte, $\mathbf{1 0}^{\mathbf{9}} / \mathbf{L}$ & M & Mean \pm SD & $2.5 \pm 0.8$ & $1.5 \pm 0.5$ & $-1.0 \pm 0.2$ \\
\hline & F & Mean \pm SD & $1.8 \pm 0.5$ & $1.0 \pm 0.4$ & $-0.8 \pm 0.1$ \\
\hline
\end{tabular}

Table 1: White Blood Cell Count Changes before and one month after RAI131 Ablation.

\section{In group B}

The values were usually in normal ranges except in two patients with low pre-operative $\mathrm{Hb}$ and WBC values; none of them showed lymphopenia or became Covid19 positive (Table 2).

\begin{tabular}{|c|c|c|c|c|c|}
\hline Blood Cells & Gender & Descriptor & After RAI131 & After Covid 19 & Difference \\
\hline WBC, $\mathbf{1 0}^{9} / \mathbf{L}$ & M & Mean \pm SD & $5.6 \pm 1.1$ & $3.6 \pm 1.0$ & $-2.0 \pm 0.2$ \\
\hline & F & Mean \pm SD & $5.0 \pm 0.8$ & $2.8 \pm 0.6$ & $-2.2 \pm 0.4$ \\
\hline Lymphocyte, $\mathbf{1 0}^{\mathbf{9}} / \mathbf{L}$ & M & Mean \pm SD & $1.5 \pm 0.5$ & $0.7 \pm 0.2$ & $-0.8 \pm 0.3$ \\
\hline & F & Mean \pm SD & $1.0 \pm 0.4$ & $<0.5 \pm 0.2$ & $-0.5 \pm 0.2$ \\
\hline
\end{tabular}

Table 2: White Blood Cell Count after RAI131 Ablation Changes in Covid19 infected 3 patients. 


\section{Discussion}

This current pilot study claimed that the co-existence of RAI-131 ablation therapy in low risk differentiated cancer thyroid patients after reaching the hypothyroid status; required for their proper preparation; in pandemic such as that Covid19 disease, could be risky and negatively affects the Covid19 respiratory disease infection potentiality and its severity itself [5].

However, these results highlight the challenges that clinicians caring for thyroid cancer face in deciding on post-surgical RAI therapy. Nuclear Medicine Consultants could all agree on 'no' for RAI for patients with low-risk disease in such pandemic situations, but there was definite disagreement, even among the consultants themselves, for patients with intermediate and high risk; i.e. metastasized disease [6]. Specific circumstances from the study 10 cases that represented decision making challenges; included: an extra-thyroidal area that presented clinical uncertainty and query N1a/N1b lymph nodes, which can be confusing if not clearly stated by a surgeon or pathologist to stratify the low risk status.

\section{Conclusion}

RAI ablation therapy in thyroid cancer patients is a safe treatment modality without any serious or persistent hematological side effects; however the slightly depressed white blood cells (WBCs; especially the lymphocytes) count has been claimed resulted in increased Covid19 infection severity; furthermore, it may negatively affecting the prognosis in Covid 19 patients; we claim that it is better to defer the radioactive iodine ablation in patients with low risk differentiated thyroid cancer in current Covid19 pandemic.
So, we recommend to defer the radioactive iodine-131 ablation therapy in recently operated up on differentiated cancer thyroid; particularly in the low risk patients; if not in the intermediate risk group; whereas the high risk group; metastasized patients could be handled case by case.

\section{References}

1. (2020) Thyroid cancer and coronavirus (COVID-19). British Thyroid Association.

2. Tufano RP, Noureldine SI, Angelos P (2015) Incidental thyroid nodules and thyroid cancer: considerations before determining management. JAMA Otolaryngol Head Neck Surg 141(6): 566-572.

3. Keldsen N, Mortensen BT, Hansen HS (1990) Hematological effects from radioiodine treatment of thyroid carcinoma. Acta Oncol 29(8): 1035-1039.

4. Sacks W, Wong RM, Bresee C, Braunstein GD (2015) Use of evidence based guidelines reduces radioactive iodine treatment in patients with low-risk differentiated thyroid cancer. Thyroid 25(4): 377-385.

5. Verburg FA, Mader U, Reiners C, Hanscheid H (2014) Long-term survival in differentiated thyroid cancer is worse after low-activity initial post-surgical 131I therapy in both high-and low-risk patients. J Clin Endocrinol Metab 99(12): 4487-4496.

6. Cooper DS, Doherty GM, Haugen BR, Kloos RT, Lee SL, et al. (2009) Revised American Thyroid Association management guidelines for patients with thyroid nodules and differentiated thyroid cancer. Thyroid 19(11): 1167-1214. 